\title{
Auftragsklärung im Coaching - eine Multi-Stakeholder-Perspektive
}

\author{
Annika Squarra ${ }^{1}$ Thomas Webers ${ }^{1,2}$ (D) \\ Online publiziert: 6. November 2020 \\ (c) Der/die Autor(en) 2020
}

\section{Zusammenfassung}

Dem Personalentwicklungsinstrument Coaching wird im Unternehmenskontext eine immer größere Bedeutung zugeschrieben. Dabei wird jedem Einsatz eine Auftragsklärung vorgeschaltet, in der verschiedene Stakeholder - Coach, Auftraggeber*In und Klient*In - involviert sind. Hierbei handelt es sich um eine Verhandlungssituation, die einen Nährboden für Konflikte bieten kann. Strategien der Stakeholder sowie ihr subjektives Erleben stellen in diesem Zusammenhang eine empirische Forschungslücke dar und werden in dieser qualitativen Interviewstudie mit Expert*Innen untersucht. Aufgrund der Vielfalt an betrachteten Aspekten ergibt sich aus den Daten der Analyse keine eindeutige Kernstrategie, darüber hinaus sind die Ergebnisse teils widersprüchlich. Die Coaches nehmen gemäß eigener Aussage eine vorwiegend parteiische Haltung gegenüber dem*der Klient*In ein und betrachten den*die Auftraggeber*In als Gegenspieler*In in der betrieblichen Arena. Die Auftraggebenden ihrerseits äußern, in der Interaktion mit Coach und Klient*In als wohlwollende Teamplayer zu agieren. Die Klient*Innen differenzieren zwischen diesen Darstellungen von Coaches und Auftraggebenden und ihren eigenen Wahrnehmungen. Es eröffnen sich letztlich Interpretationsspielräume, sodass weiterer Forschungsbedarf offensichtlich wird.

Schlüsselwörter Arbeitsfähigkeit · Auftragsklärung · Setting · Verhaltensprävention · Verhältnisprävention · Verhandlung

\section{Contract Clarification in Coaching-a Multi-stakeholder-perspective}

\begin{abstract}
The personnel development instrument of coaching is becoming increasingly important in the corporate context. Each assignment is preceded by a contract clarification, in which various stakeholders—coach, employer and client—are involved. This is a negotiation situation that can provide a breeding ground for conflicts. Strategies of stakeholders as well as their subjective experience represent an empirical research gap in this context and are examined in this qualitative interview study with experts. Due to the variety of aspects considered, the data of the analysis does not provide a clear core strategy; furthermore, the results are partly contradictory. According to their own statements, the coaches take a predominantly biased attitude towards the client and regard the employer as an opponent in the operational arena. For their part, the employers express that they act as benevolent team players in their interaction with the coach and client. Clients differentiate between these representations of coaches and clients and their own perceptions. Ultimately, this opens up scope for interpretation, so that further research needs become apparent.
\end{abstract}

Keywords Behavioral Prevention · Conditional Prevention · Contract clarification · Negotiation · Setting · Work Ability

\section{Einleitung}

$\triangle$ Annika Squarra

ASquarra@gmx.de

1 Psychology School, Hochschule Fresenius, Köln, Deutschland

2 FOM-Hochschule, Bonn, Deutschland
Menschen unterscheiden sich in ihrem Umgang mit Krisensituationen, aber viele haben im privaten sowie beruflichen Kontext früher oder später das Bedürfnis, Unterstützung zu erfahren. Aus diesem Grund bieten mittlerweile viele Unternehmen Coaching für ihre Mitarbeiter*Innen an. Ein 
noch wenig erforschter Bereich stellt in diesem Zusammenhang die Auftragsklärung dar. Während diese im privaten Coaching lediglich zwei Akteur*Innen betrifft, Coach und Klient*In (Zweiersetting), kommt im Unternehmenskontext ein*e weitere*r Akteur*In hinzu: der*die betriebliche Auftraggeber*In (Dreiersetting). Da das Unternehmen Coaching einkauft, spielen dessen*deren Interessen zwangsläufig eine Rolle (Schwertl 2015). Die Auftragsklärung im Dreiersetting gestaltet sich allerdings komplexer als auch dynamischer im Vergleich zum Zweiersetting: Es scheint eher wahrscheinlicher als selten, dass sich Unstimmigkeiten ergeben können. Wie verhalten sich die Beteiligten in solchen schwierigen Situationen und was erleben sie dabei? Dieser Fragestellung der Auftragsklärung im Dreiersetting, widmet sich die hier präsentierte Studie.

\section{Theoretischer Rahmen}

Der Ablauf der Auftragsklärung im Coaching ist empirisch bislang weitgehend unerforscht. Es finden sich in der Literatur überwiegend kursorische Betrachtungen anhand von Fallvignetten (Pühl 2006, 2008; Löwer-Hirsch 2017). Dieses Desiderat wiegt umso schwerer, da die Diskussion um die Definition von Coaching weiterhin kontrovers geführt wird und zugleich die Anforderungen an Coaching heterogen sind (Greif et al. 2018). Eine Gefahr besteht somit offensichtlich darin, Coaching für ungeeignete Fragestellungen in Anschlag zu bringen. Greif und Bertino (2018) exemplifizieren dies am Beispiel von Burnout eindrücklich und zeigen Grenzen für Coaching zur Psychotherapie auf. Zudem kritisieren sie, dass zur Burnout-Prävention im populären Quellen überwiegend Interventionen der Verhaltensprävention genannt werden.

\subsection{Arbeit und Gesundheit}

In der Arbeitspsychologie gehört es seit Langem zum anerkannten Wissenstand, dass erfolgreiches und gesundheitsförderliches Arbeiten von drei sich wechselseitig bedingenden Domänen determiniert wird: Dem*der Mitarbeiter*In, dessen*deren Gesundheit und Kompetenzen, der Arbeitsgestaltung und der Führung. Ulich (2011) leitet diesen Erkenntnisstand (MTO-Konzept: Mensch - Technik - Organisation) historisch her. Seit den 1990er-Jahren hat sich der Fokus, angeregt durch die Erkenntnisse der Forschungsgruppe um Ilmarinen, Tuomi \& Seitsamo (2005), stärker in Richtung gesundheitsförderliches Arbeiten verschoben und verdichtet sich im Konzept Work Ability (Arbeitsfähigkeit) (s. Abb. 1), das sich auch in Deutschland breiter Aufmerksamkeit, bspw. im Rahmen der Initiative Neue Qualität der Arbeit (INQA), erfreut. „Das Konzept der Arbeitsfähigkeit ist ein Modell, welches verhaltens- und verhältnisbezogene

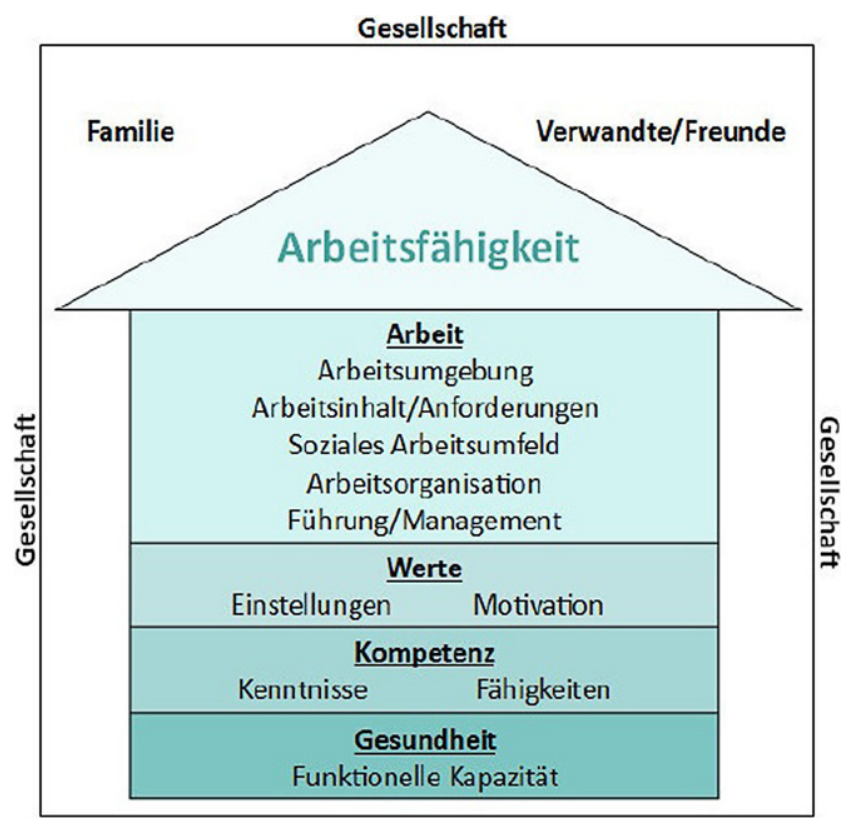

Abb. 1 Haus der Arbeitsfähigkeit (WAI-Netzwerk 2015, S. 7; modifiziert nach Ilmarinen, Tuomi \& Seitsamo 2005)

Ansätze für nachhaltige Förderung der Arbeitsfähigkeit integriert. Diese erfordert regelmäßig Präventionsmaßnahmen in den vier Handlungsfeldern: (1) individuelle Gesundheit, (2) professionelle Kompetenz der Arbeitnehmer, (3) Arbeitsumgebung und (4) Arbeitsorganisation und Führung“ (WAI-Netzwerk 2015, S. 8).

Doch dieser Erkenntnisstand scheint in den Betrieben noch nicht in der Breite berücksichtigt zu werden, bilanziert die wissenschaftliche Standortbestimmung zur psychischen Gesundheit in der Arbeitswelt (BAuA 2017). Einerseits beklagt sie verbreitete Informationsdefizite. So würde psychische Belastung oft als psychische Erkrankung oder Störung missverstanden. Andererseits würden Hilfsangebote von Präventionsfachleuten pauschal abgewehrt, da ,sehr schnell Aspekte des betrieblichen Interessengefüges und der jeweiligen Organisationskultur berührt sind, die der Herausbildung eines geteilten Problemverständnisses entgegenstehen können und sich u.U. stark gegen Veränderungen sperren“" (BAuA 2017, S. 99).

Eine Gefahr besteht offenbar darin, in den Betrieben kritische Problemstellungen einseitig personal zu attribuieren und primär verhaltenspräventiv anzugehen, bspw. mit (Gesundheits-)Coaching (Bamberg und Vincent-Höper 2018). Das Ziel lautet dann verkürzt: Verbesserung der Selbststeuerung des*der Mitarbeiter*In. In der Literatur kritisieren etliche Coaches solche Aufträge dezidiert: „(...) dass Coaching nur erfolgreich sein kann, wenn Persönlichkeits- und Systementwicklung im Gleichschritt verlaufen. Ansonsten läuft Coaching Gefahr, degradiert zu werden: Wir sollen dann flächendeckend die Schnittstellen coachen, die im Change-Prozess unsauber durchdacht wor- 
den sind und die jetzt leiden, aufbegehren oder was auch immer" (Fatzer und Webers 2008, S. 13). Zudem scheint sich ein ansteigender Trend zur Förderung der Resilienz im Arbeitskontext, insbesondere im betrieblichen Coaching, abzuzeichnen. Generell darf die Verfolgung solch individualisierter Ansätze die Auswirkungen von Belastungen auf organisationaler Ebene nicht systematisch ausklammeren (Limmer und Schütz 2018). Auch Klient*Innen erleben offensichtlich als zweithäufigsten negativen Effekt von Coaching $(17,1 \%)$, dass , die ursprünglichen Ziele (...) abgewandelt [wurden], ohne dass er das wollte" (Schermuly 2016, S. 209).

Der Einfluss von Führung auf das Belastungserleben von Mitarbeitenden ist inzwischen, so zeigen einige Metaanalysen (Montano et al. 2017; Schyns und Schilling 2013), gut erforscht. Gleiches gilt für den Einfluss von Arbeitsbedingungen (Franke et al. 2015; Wieland et al. 2009), sodass der verhältnispräventive Ansatz bei der Betrachtung kritischer betrieblicher Problemstellungen mindestens gleichwertig verfolgt werden muss. Und so folgern auch Bamberg und Vincent-Höper für Coaching: ,(...) dass der gesundheitsförderliche Effekt am höchsten ist, wenn man gleichzeitig sowohl an der Bedingungsebene als auch an der Personenebene ansetzt" (Bamberg und Vincent-Höper 2018, S. 239).

\subsection{Die Auftragsklärung im Coaching}

Das Thema Auftragsklärung steht am Anfang jedes Coaching-Prozesses. Es handelt sich um eine sensible Phase, da zunächst abgeklärt werden muss, ob Coaching überhaupt die richtige Maßnahme darstellt. Fischer, von Schlippe \& Borst (2014) differenzieren vier Fragen, die vor der konkreten Arbeit mit dem*der Klient*In zu beantworten sind:

1. „Anlass: Habe ich verstanden, was mein Gegenüber (...) zu mir bringt (...)?

2. Anliegen: Habe ich verstanden, was er (und bedeutsame Dritte) mit der gemeinsamen Arbeit erreichen möchte, was er wünscht/erwartet/befürchtet (...)?

3. Auftrag: Habe ich verstanden, was er genau von mir will (...)?

4. Abmachung: Kann ich mir vorstellen, mit dem, was er möchte, mitzugehen? Wozu bin ich bereit, wozu nicht? Wie kann ich wertschätzend mit dem umgehen, zu dem ich nicht bereit bin, welche Alternative kann ich anbieten - oder die Kooperation freundlich beenden? (...)“ (Fischer, von Schlippe \& Borst 2014, S. 262; Hervorhebungen i. O.).

Die Auftragsklärung im Coaching ist allerdings keine Angelegenheit, die man zu Beginn abschließend klären kann, sondern sie begleitet alle Beteiligten durch den Coaching-Prozess: „Die Worte ,Auftrag“ wie ,Auftragsklä- rung““, so erläutern die Autor*Innen, „suggerieren eine missverständliche Klarheit, die am Anfang überhaupt nicht und am Ende der Klärung auch nicht vollständig gegeben ist. Wir haben es also generell mit Vagem, mit Ungefährem zu tun (...). Je mehr man hinter die Kulissen des Auftrages blickt, umso mehr werden ganze ,Erwartungspakete sichtbar. Solche Aufträge erweisen sich als MatroschkaPuppen“ (Fischer, von Schlippe \& Borst 2014, S. 260).

Von diversen Autor*Innen werden Vorschläge zur Abgrenzung (Indikation) unterbreitet: So grenzt bspw. Rauen (2014) Coaching von Psychotherapie, Supervision, Mentoring, Beratung und Training ab. Solcherlei Abgrenzungen sind einerseits sinnvoll, um professionelle Fehler zu vermeiden: Psychotherapie steht in Deutschland gesetzlich unter Erlaubnisvorbehalt. Andererseits erweisen sich solche Abgrenzungsversuche oft als oberflächlich marketinggetrieben und einer argumentativen Diskussion kaum standhaltend (Webers und Zickermann 2019). Zudem verwischen sich in der Praxis leicht die Domänengrenzen, ob aus Unkenntnis oder aus bewusstem Kalkül (Werner und Webers 2016). Bei der Auftragsklärung in der Praxis sollten solche Aspekte virulent und bestmöglich bearbeitet werden.

Eng verbunden mit dieser Ausgangslage ist das Thema Rollenklärung (Kühl 2018). Es ist für Coach und Klient*In unabdingbar, die gegenseitigen Erwartungshaltungen zu kennen und zu klären, ob man sich darauf einstellen möchte (Veith und Veith 2014): Was erwartet der*die Klient*In vom Coach? Wer will der Coach für den*die Klient*In sein? Und welche Implikationen sind damit verbunden? Die Vorstellungen der Beteiligten können unterschiedlich und sogar inkompatibel sein. Das sog. Funktionspendel (Wolff 2012) grenzt Coaching von der Therapie einerseits und von der Expertenberatung andererseits ab. Wenn sich diese Unterscheidung zwischen Coaching und Therapie bzw. Expertenberatung auch nicht trennscharf vornehmen lässt, so verbleibt doch die Beziehungsdefinition - Dialog auf Augenhöhe - als Postulat für das Coaching. Indem Coaching zudem als Koproduktion definiert wird, werden Fremdhilfeerwartungen relativiert. Diese, von professioneller Seite gesetzten Bedingungen müssen dem*r Klient*In nicht bekannt sein, und er*sie kann sie als Voraussetzungen auch ablehnen. Insofern handelt es sich bei der Auftragsklärung um eine Verhandlungssituation - wie schon Fischer, von Schlippe \& Borst (2014) darlegen.

\subsection{Auftragsklärung im Dreiersetting}

Wenn das Unternehmen Coaching einkauft, sind Auftraggeber*In und Klient*In nicht dieselbe Person. Die Interessen des*der Auftraggeber*In spielen zwangsläufig eine Rolle und müssen nicht identisch mit denen des*der Klient*In sein. Der*die betriebliche Auftraggeber*In kann zudem unterschiedlich positioniert sein. Die Marburger Coa- 
ching-Marktstudie erbringt das Ergebnis, dass in nur knapp einem Viertel $(22,4 \%)$ der befragten Unternehmen Fachexpert*Innen (Personalentwickler*Innen) über die CoachingIndikation entscheiden (DBVC 2013). Häufiger sind es die Geschäftsführung $(28,4 \%)$ oder gleichauf die direkten Vorgesetzten (Abteilungs-/Bereichsleitung: 22,4\%) - kritisch betrachtet sind sie Coaching-Laien, wobei angemerkt werden muss, dass vor allem in kleinen und mittleren Unternehmen (KMU) eine solche Ausdifferenzierung der Rollen aufgrund der Unternehmensgröße oft gar nicht gegeben ist.

Im Business-Coaching wird diese Stakeholder-Kombination - bezugnehmend auf das im Rahmen der Transaktionsanalyse von English (1975) entwickelte Modell - als Dreieckskontrakt bezeichnet (Schmid und Hipp 2003; Limpächer und Limpächer 2003, 2015). Wenn der interne oder externe Coach angefragt wird, gerät er*sie in die Situation, zwei oder mehrere Kund*Innen zugleich zu haben. Somit bestehen drei bilaterale Relationen, die die Autor*Innen als Verträge bezeichnen:

- Auftraggeber*In/Mitarbeiter*In: Hier besteht ein Arbeitsvertrag und damit auch ein Direktionsrecht (Hierarchie).

- Auftraggeber*In/Coach: Hier besteht ein Dienstvertrag.

- Mitarbeiter*In/Coach: Hier besteht ein psychologischer Vertrag.

Diese drei Verträge müssen nicht harmonisieren, sondern können sich (partiell) widersprechen. Das Konzept des Dreiecksvertrags zielt darauf ab, solche Unterschiede kommunizierbar zu machen. Der bilaterale - und weiterhin vertrauliche - Coaching-Prozess wird durch Round-Table-Gespräche der Stakeholder (Coach, Klient*In, Führungskraft und ggf. Personaler*In) zu Beginn und am Ende gerahmt. In diesem Gespräch sollen bereits im Vorfeld die Vertragsbedingungen (Zielsetzung, Rahmenbedingungen) geklärt werden. „Diese (...) als Vertragsarbeit bezeichnete Auftragsklärung soll die Rollen, Erwartungen und Interessen aller Prozessbeteiligten transparent machen" (Limpächer und Limpächer 2003, S. 62). Die Autor*Innen beschreiben die Interessen der beteiligten Stakeholder folgendermaßen:

- Die Führungskraft vertritt qua Rolle die Ziele der Organisation des*der Klient*In. Sie soll „unangenehme Führungsaufgaben nicht auf einen ,Externen“ abwälzen“ (Limpächer und Limpächer 2003, S. 64).

- Der Coach hat als externe*r Expert*In die Möglichkeit, Stellung zu nehmen, welche Anliegen und Erwartungen sich tatsächlich mittels Coaching realisieren lassen. Er*sie kann die Interaktion zwischen dem*der Klient*In und dessen*deren Vorgesetzten direkt beobachten und erhält dadurch wertvolle systemische Einblicke.
- Der*die Klient*In erfährt die Gründe, die seine*ihre Führungskraft und der*die Personaler*In für den Coaching-Auftrag anführen und kann seine*ihre eigene Sichtweise aktiv einbringen. Durch das gemeinsame Festschreiben der Coaching-Ziele wird Klarheit, Sicherheit und Zielerreichungsmotivation freigesetzt.

- Der*die Personaler*In vertritt die Interessen des gesamten Unternehmens, die durchaus mit den Partialinteressen einer Abteilung in Konkurrenz stehen können. Als Prozesseigner*In steuert er*sie den gesamten CoachingProzess. Tendenziell übernimmt er auch die Rolle eines*einer „Anwalts*Anwältin“ des*der Mitarbeiter*In. Denn das Unternehmen als Ganzes mag - bspw. im Rahmen der Personalentwicklung - weitere Interessen mit dem*der Mitarbeiter*In verbinden als aus der Perspektive der Abteilung aufscheinen, in der der*die Mitarbeit*In derzeit tätig ist.

Sowohl Limpächer und Limpächer (2003) als auch Schmid und Hipp (2003) sprechen im Zusammenhang des Dreiecksvertrags das Thema Führung an, der*die Vorgesetzte soll seine*ihre Führungsverantwortung nicht an den Coach delegieren. Schmid und Hipp präsentieren bemerkenswerterweise eine stark verhaltenspräventiv gefärbte Fallgeschichte - die Problemursachen werden also deutlich auf den*die Klient*In attribuiert. Das Thema Arbeitsgestaltung wird von keiner Autor*Innengruppe dezidiert thematisiert. Schmid und Hipp warnen immerhin vor Fallen bei der Kontraktgestaltung: „Kommt es zu Schieflagen in der Kontraktgestaltung, etwa indem sich ein Coach mit dem Klienten solidarisiert, besteht die Gefahr, dass der Kontrakt mit dem Auftraggeber aus dem Blick gerät" (Schmid und Hipp 2003, S. 257).

\subsection{Organisationskultur als Deutungsrahmen}

In einer viel beachteten empirischen Untersuchung (Bachmann 2016) zu impliziten Theorien über Lernen und Veränderungen durch Coaching in Organisationen werden die Implementierungsformen von Coaching sowie Vorstellungen über Coaching erhoben. Personalverantwortliche aus Unternehmen und Institutionen in Deutschland äußern sich in der Studie zu Coaching für Personen mit Führungsverantwortung. In Organisationen lassen sich demnach vier prototypische Coaching-Verständnisarten unterscheiden, die sich durch die beiden Dimensionen Person vs. Organisation sowie Autonomie vs. Steuerung ergeben. Bachmann (2016) betont, dass in der Darstellung der von ihm gefundenen Klassen keine Wertung enthalten sei, sondern lediglich der Status quo beschrieben werde:

- Therapie/Heilung. Primär personenfokussiert, man möchte den Mitarbeitenden etwas Gutes tun. Typische 
Themen: Work-Life-Balance, Zeit- und Selbstmanagement. Es herrscht eine gewisse Ergebnisoffenheit (Autonomie).

- Selbstorganisation/Empowerment. Primär organisationsfokussiert, man möchte die Zusammenarbeit verbessern. Typische Themen: Führung und Rollenklärung, Potenzialentwicklung. Es herrscht eine gewisse Ergebnisoffenheit (Autonomie).

- Expert*Innenberatung/Optimierung. Primär organisationsfokussiert, der „Laden soll laufen“. Typische Themen: Konflikte, Teamprobleme, Mikropolitik und Entscheidungen. Es herrscht eine deutliche Ergebniserwartung (Steuerung).

- Pädagogik/Erziehung. Primär personenfokussiert, man möchte Defizite der Mitarbeitenden bearbeiten. Typische Themen: Standing und Auftreten. Es herrscht eine deutliche Ergebniserwartung (Steuerung).

Für die eigene Untersuchung relevant ist die Erkenntnis, dass weniger von einer einheitlichen Coaching-Vorstellung der Auftraggebenden ausgegangen werden darf, sondern die Strategien von Unternehmen offenbar differenziert zu betrachten sind. „Das Verständnis von Coaching hat Einfluss auf die Auswahlkriterien für Coaches, die Coachingthemen, die Erwartungen an Coaching und die dem Coaching zugeschriebenen Funktionen für die Organisation und ihre Mitglieder. Auch einige Prozesscharakteristika, wie die Gestaltung der Auftragsklärungsgespräche, die Arbeit mit Zielvereinbarungen sowie die Evaluation und Dokumentation, werden dadurch beeinflusst" (Bachmann 2016, S. 251). So neigen Unternehmen mit einem eher pädagogischen CoachingVerständnis dazu, den Auftrag zunächst zwischen Coach und Personaler*In oder Führungskraft zu klären und erst im Anschluss den*die Klient*In einzubeziehen. In der Gruppe „Expert*Innenberatung“ hingegen erfolgt das Erstgespräch häufiger in der Form eines Dreieckskontraktgesprächs (Klient*In, Vorgesetzte*r und Personaler*In - ohne Coach).

Bezogen auf das Konzept Work Ability (Arbeitsfähigkeit) ergeben sich an dieser Stelle mehrere Fragen: Zunächst ist zu konstatieren, dass zwei von vier Typen personenbezogen sind. Folglich könnte man vermuten, dass im Coaching-Verständnis der betrieblichen Auftraggebenden diese individualisierende Attribution überrepräsentiert ist. Andererseits dominiert bei zwei von vier Typen eine kontrollierende Absicht des Unternehmens. Die Bereiche Führung und Organisationsgestaltung als Ansatzpunkte im Konzept Work Ability werden in der Expert*Innenberatungskultur ausgeklammert. Dies mag man als der Natur der Sache angemessen betrachten (Direktionsrecht). Andererseits kann so Verbesserungspotenzial leichtfertig ignoriert werden.

\subsection{Dreiecksverträge als soziales Dilemma}

Schon Dehner (2013) hat - ohne allerdings die von Bachmann (2016) vorgenommene Typisierung vorweg zu nehmen - das Konzept Dreieckskontrakt kritisiert. Er zweifelt an, dass sich in der Praxis klare Vereinbarungen mit allen Beteiligten treffen lassen: „Weder wird der Auftraggeber im Klartext sagen, was er über den Klienten denkt, noch wird der Klient eindeutig Stellung beziehen, wenn ihm das im Angesicht seines Chefs zu riskant erscheint. So entgehen dem Coach unter Umständen wichtige Informationen“ (Dehner 2013, S. 35). Durch das bestehende Hierarchiegefälle (Gesichtsverlust) werden die Themen Führung und Arbeitsgestaltung folglich tendenziell ausgeblendet.

Schwertl (2015) beschreibt das Dilemma zweier Verträge und unzulänglicher Klärung dieser Lage für den Coach wie folgt: „Die grundsätzliche Alternative (der Coach als Komplize der Neugier des Arbeitgebers versus der Coach als verschwiegener Beichtvater) greift zu kurz und produziert in beiden Fällen Ergebnisse, die hinter den Möglichkeiten zurückbleiben. Besser ist es, wenn sich der Coach als ,Dialogförderer für das ganze System ‘ anbietet" (Schwertl 2015, S. 50). Der Autor nennt es eine Frage der Haltung, Transparenz herzustellen und auf die Macht der Kommunikation zu bauen, um nicht nur unerfüllbaren Aufträgen vorzubeugen, sondern das Beste für alle Beteiligten zu ermöglichen.

\section{Methodik}

Trotz vorhandenen Wissens, wie Auftragsklärungen in der Theorie durchgeführt werden sollten und welche Aspekte es zu beachten gilt, existiert bisher kein empirisches Wissen über Formen des tatsächlichen Ablaufs. Das genaue Verhalten und Erleben der Beteiligten in der Praxis gilt als unbekannt und weist somit eine Forschungslücke auf. Dies wirft insbesondere die Frage auf, wie schwierige Verhandlungssituationen in der Auftragsklärung konkret gelagert sind.

\subsection{Generierung der Forschungsfrage}

Aufgrund immer wieder geäußerter Kritik an und aus der Coaching-Praxis lässt sich die Hypothese aufstellen, Coaching wird in den Betrieben überwiegend als individualisierte Problemlösungsstrategie verstanden, ggf. sogar instrumentalisiert. Wenn solche individualisierenden Diagnosen - es liegt am*an der Mitarbeiter*In - oft mit Wahrheitsanspruch und Positionsmacht kommuniziert werden, immunisiert sich der*die Diagnosesteller*In, ob nun gut gemeint oder vorsätzlich, gegen Kritik und wartet in einer Art Konsumentenhaltung auf den*die „reparierte*n“ Mitarbeiter*In. Es leuchtet unmittelbar ein, dass ein Infrage 
stellen solcher einseitigen und selbsterstellten Diagnosen von Auftraggebenden seitens des Coachs riskant ist - insbesondere in steuerungsdominanten Kulturen. Daher stellt sich die Frage, wie, also mit welchen Taktiken, Coaches auf solche Konstellationen reagieren, und ob und wie diese für die Erbringung der Dienstleistung Coaching funktional sind.

Damit ergibt sich eine weitere Hypothese: Einseitig beschränkt praktiziert steht Coaching in Unternehmen tendenziell in der Gefahr, suboptimale Prozesse und Ergebnisse zu produzieren, da ein auf das Individuum enggeführtes Verständnis Problemlagen in den Bereichen Arbeitsgestaltung und Führung nicht oder lediglich partiell bearbeiten kann. Als leitende Forschungsfrage wird daher generiert: Welche Strategien verfolgen Coaches, Auftraggebende und Klient*Innen in schwierigen Situationen bei der Auftragsklärung und wie erleben sie diese? Im Besonderen werden folgende speziellen Fragen aufgeworfen:

- Ist anzunehmen, dass Coaching auch für unpassende Problemlagen eingesetzt wird, und wie ließe sich das erklären?

- Ist eine Tendenz erkennbar, Coaching eher verhaltensstatt verhältnispräventiv zu verstehen und zu operationalisieren, und wie ließe sich das erklären?

- Ist die Gestaltung der Auftragsklärungsform abhängig vom Organisationskulturtyp?

- Bestehen deutliche Interessensunterschiede zwischen den Stakeholdern?

\subsection{Untersuchungsdesign}

Der Ablauf der Auftragsklärung im Coaching ist bislang empirisch weitgehend unerforscht. Dadurch liegen keine Daten über schwierige Situationen vor, was den Einsatz des Expert*Inneninterviews als Untersuchungsinstrument begründet: Es zielt darauf $a b$, schwer zugängliche Informationen bestimmter Bereiche in Form von Rollenwissen der Expert*Innen zu gewinnen (Helfferich 2014). Deren Expertise soll Aufschluss über Verhaltens- und Erlebensweisen geben und damit den theoretischen Forschungsstand empirisch erhellen und ergänzen.

Im Rahmen der vorliegenden Studie wird daher ein qualitatives Forschungsdesign mit halbstrukturierten Interviews verwendet. Durch eine Multi-Stakeholder-Perspektive (Coach - betriebliche*r Auftraggeber*In - Klient*In) soll Wissen gewonnen und gleichzeitig sollen Widersprüche in Verhalten und Erleben der Akteur*Innen sichtbar gemacht werden. In diesem Zusammenhang soll ebenfalls herausgearbeitet werden, welche Einschätzungen, Wahrnehmungen und Emotionen die Stakeholder erleben. Dies soll zu einer Bereicherung empirisch fundierter Erkenntnisse zur Auftragsklärung im Unternehmenskontext beitragen.
Die leitfadengestützten Expert*Inneninterviews haben eine jeweilige Länge von ca. 30 bis $80 \mathrm{~min}$. Die Interviews finden im Face-to-Face-Setting oder telefonisch statt und werden anschließend transkribiert sowie inhaltsanalytisch nach Mayring (2015) ausgewertet. Die Fragenblöcke sowie die darin enthaltenen Leitfragen des Leitfadens sind inhaltlich für jede Gruppe nahezu identisch, jedoch perspektivisch auf die jeweilige Untergruppe angepasst. Um eine bessere Vergleichbarkeit der Antworten zu erzielen, wird den Teilnehmenden zu Beginn des Interviews eine fiktive Ausgangssituation präsentiert, in welcher ein*e unter Stress leidende*r Mitarbeiter*In ein Coaching bekommen soll. Dieser Fokus dramatisiert die Situation der Auftragsklärung einerseits, andererseits eignet sich diese eher diffuse Diagnose in besonderer Weise dazu, verhaltenssowie verhältnispräventive Erlebens-, Bewertungs- und Handlungsweisen zu untersuchen (Bamberg und VincentHöper 2018).

\subsection{Stichprobe}

Die Stichprobe $(N=18)$ der Untersuchung wird in drei Gruppen eingeteilt ( $3 \times 6$-Design), sodass alle Expert*Innengruppen (Coaches, Auftraggebende und Klienten*Innen) gleichstark vertreten sind. Dabei werden die Interviewteilnehmenden im Sinne einer selbstgenerierten Stichprobe überwiegend durch Aufrufe in Newslettern, Forumsbeiträgen diverser Coaching-Verbände und in den sozialen Medien (Xing, LinkedIn) akquiriert. Der Großteil der Klient*Innen wird durch das Schneeballverfahren gewonnen, also durch Weiterempfehlungen bereits interviewter Coaches akquiriert.

In der Unterstichprobe der Coaches sind alle Teilnehmenden weiblich. Der Altersdurchschnitt beträgt 51,2 Jahre und die durchschnittliche Berufserfahrung liegt bei 26 Jahren. 16,3 Jahre stellt die durchschnittliche Berufserfahrung in der Tätigkeit als Coach dar, ein Drittel der Coaches haben weniger und Zweidrittel mehr als zehn Jahre Erfahrung. Als homogene Merkmalsausprägungen werden ein Studienabschluss und eine zertifizierte Coaching-Weiterbildung ermittelt sowie eine Tätigkeit als externer Coach.

Die Unterstichprobe der Auftraggebenden kann zunächst in drei Führungskräfte und drei Personaler*Innen unterteilt werden. Von diesen sind vier männlich und zwei weiblich, sämtliche Führungskräfte gehören dem männlichen Geschlecht an. Das Durchschnittsalter beträgt 50,3 Jahre, der*die jüngste Auftraggeber*In ist 37 und der*die älteste 62 Jahre alt. Die allgemeine durchschnittliche Berufserfahrung liegt bei 25,1 Jahren, in den jeweiligen Positionen als Personaler*In oder Führungskraft bei 18,3 Jahren.

In der Unterstichprobe der Klient*innen sind drei männliche und drei weibliche Teilnehmende vertreten. Der Altersdurchschnitt beträgt hier 43,3 Jahre, der*die jüngste Kli- 
ent*In ist 25 Jahre und der*die älteste 60 Jahre alt. Darüber hinaus verfügen die Klient*Innen im Durchschnitt über 19,3 Jahre Berufserfahrung.

\subsection{Datenerhebung und -auswertung}

Alle Interviews werden mit einem Aufnahmegerät aufgezeichnet und anschließend mithilfe des Transkriptionsprogramms f5Transkript transkribiert. Dabei werden die Regelsysteme zur Transkription von Dresing und Pehl (2015) verwendet. Die qualitative Inhaltsanalyse nach Mayring (2015) wird als Analysemethode gewählt. Zur Umsetzung wird die QDA-Software f5Analyse für die inhaltsanalytische Auswertung eingesetzt.

Die Erhebung ergibt ein Textkonvolut von 339 Seiten. Die Inhaltsanalyse als datenreduzierendes Verfahren hat das Ziel, das für die Forschungsfrage relevante Material zu selektieren und zu komprimieren - bei gleichzeitiger Wahrung der inhaltlichen Güte (Mayring 2015). Daher wird die induktive Kategorienbildung als Ablaufmodell, welche der Methode der Zusammenfassung angehört, als Analysetechnik ausgewählt. Die Kodierung des Materials erfolgt durch die Autorin.

\section{Ergebnisse}

Zunächst werden die Strategien der einzelnen Stakeholder separat betrachtet. Es schließt sich eine deskriptiv vergleichende Betrachtung an.

\subsection{Strategien der Coaches}

\subsubsection{Fokus: Verhaltens- vs. Verhältnisprävention}

Die bei der Diagnose „Mitarbeiter*In im Stress“ primäre Frage betrifft eine psychotherapeutische Indikation. Die führende Strategie der Coaches ist, diesen Bedarf - mit Verweis auf ihre Lebenserfahrung - durch Vorgespräche mit dem*der Klient*In zu entscheiden. Als persönliche Voraussetzungen aufseiten der Klient*Innen benennen die Coaches die Wahrnehmung einer konstruktiven Arbeitshaltung sowie einer positiven Arbeitsbeziehung als relevant. Einen vom*von der Auftraggeber*In ausdrücklich formulierten Wunsch, den*die Klient*In resilienter bezüglich Stress zu machen, würden die Coaches in der Mehrzahl deutlich abwehren. Stattdessen beziehen sie Kontextvariablen allgemein, insbesondere aber die Arbeitsumgebung und die Führungssituation, in ihre Problemdiagnose mit ein. Die Konsequenzen bezüglich Arbeitsumgebung sind allerdings heterogen und eher defensiver Art: Manche Coaches fühlen sich dann grundsätzlich nicht mehr zuständig: „Auf der Ebene 1, individuelles Verhalten, arbeitet der
Coach, auf der Ebene 2 und 3 arbeitet er irgendwie nicht mehr (...). Das heißt, ich kann als Coach sehr wohl erkennen: Wir haben hier nicht nur ein Verhaltensthema, sondern auch ein prozessuales Thema, aber ich kann das nicht bearbeiten an der Stelle“ (Squarra 2019, C 4, S. 95). Andere verweisen darauf, dass man den*die Klient*In durch das Coaching stärken könne, das Thema in Zukunft besser anzugehen: „(...) oder ihn dazu anleite, dass er überlegt, welche Möglichkeiten er hat, die Situation zu verändern. Und da gibt es so einen schönen Spruch: Love it (...), change it or leave it (...)“ (Squarra 2019, C 5, S. 115). Das offene Gespräch mit der Führungskraft über diesen Umstand wird von Coaches eher nicht gesucht. Sollte allerdings Führung als Thema dominant werden, erklären die Coaches mehrheitlich, das Gespräch mit der Führungskraft - trotz vermuteter geringer Erfolgsaussichten - zu suchen: „Habe ich schon vorsichtig angesprochen, ist aber in der Regel nicht sehr häufig von Erfolg gekrönt“ (Squarra 2019, C 6, S. 135). Die Gesprächsführung wird daher nicht klar und damit konfrontativ, sondern bewusst ,weich“ gestaltet mit dem Ziel, die Führungskraft zum Perspektivenwechsel einzuladen. Zugleich agieren die Coaches in Richtung Empowerment der Klient*Innen, damit diese Führungsthemen von sich aus ansprechen, um Veränderungen zu bewirken. Als Ultima Ratio werden Mediation oder ein konstruktiver Haltungswechsel der Klient*Innen benannt.

\subsubsection{Auftragsklärung als Verhandlungssituation}

Sechs Varianten der Auftragsklärung berichten Coaches. Es dominieren zwei Formen: die ausschließlich bilaterale Klärung zwischen dem Coach und dem*der Klient*In sowie ein sukzessives Modell, indem dieser eine Auftragsklärung mit der Führungskraft oder dem*der Personaler*In vorausgeht. Lediglich ein Coach berichtet, dass es keine Klärung mit dem*der Klient*In, sondern nur eine mit dem*der Auftraggeber*In gibt. Die anderen bevorzugen die bilaterale Variante und befürchten eher Instrumentalisierungsversuche in der sukzessiven Form.

Oberstes Anliegen der Coaches bei der Auftragsklärung ist die Absicherung eines diskreten Coaching-Prozesses. Sie wollen ein Hineinregieren von Führungskräften verhindern. „Viele Führungskräfte besuchen Coaching-Wochenendkurse, und dann denken sie, sie können das. Egal, wo ich bin, ich sage ,Ich bin Coach', sagen drei sofort ,Ich auch!‘. (...) Die Gefahr (...) ist, dass ungelöste Führungsprobleme im Coaching gelöst werden sollen. Ich soll den Mitarbeiter passend machen. Das will ich gar nicht" (Squarra 2019, C 3, S. 77).

Alle Coaches berichten, dass sie mit dem*der Auftraggeber*In einen schriftlichen Vertrag (Formalia) schließen. Darüber hinaus schließen fünf von sechs Coaches mit ihrem*ihrer Klient*In einen mündlichen Vertrag, der das The- 
ma und die grobe Zielrichtung beinhaltet. Sollte sich eine Inkongruenz zwischen der Zielvorstellung des*der Auftraggeber*In und dem*der Klient*In offenbaren, berichten alle Coaches, eindeutig Partei für den*die Klient*In zu ergreifen. Ein Drittel der Befragten erklärt, man würde das Coaching sogar abbrechen oder an den Zielen des*der Auftraggeber*In vorbei coachen: „Das bedeutet, dass ich als Coach mit ihm erarbeite, wie kann er denn da (...) ExitStrategien entwickeln? (...). Das werde ich der Personalabteilung aber erstmal überhaupt nicht sagen. Ja, an der Stelle driftet das auseinander, ganz klar" (Squarra 2019, C 4, S. 101). Ansonsten würden mehrheitlich individualisierende Diagnosen der Auftraggebenden infrage gestellt, im Schutz der diskreten Coaching-Situation eine eigene Diagnostik betrieben und dazu passende Interventionen angeschlossen: ,....) das kläre ich nur so grob mit dem Personaler, aber dann intensiv mit dem Coachee und letztendlich ist das, was der sagt, ausschlaggebend (...)“ (Squarra 2019, C 6, S. 134). Wahrgenommene Delegationsversuche von Führungsverantwortung würden alle Coaches mit den Führungskräften besprechen und zurückweisen. Eine abschließende Rückmeldung von Ergebnissen an den*die Auftraggeber*In durch den Coach erfolgt entweder gar nicht, durch den*die Klient*In selbst oder lediglich auf einer sehr abstrakten Ebene. „Nur das, was der Coachee mir erlaubt, ich würde gar keine Informationen weitergeben, die der Coachee für mich nicht freigegeben hat" (Squarra 2019, C 1, S. 34).

\subsubsection{Der Round-Table: Auftragsklärung im Dreieckskontrakt}

Im Round-Table-Gespräch berichten die Coaches überwiegend, ließen sich konkrete Ziele mangels Offenheit nicht vereinbaren. ,(..), wenn ich an Zielformulierung denke (...), das kann nicht als Jahrmarktsveranstaltung in dieser ViererRunde stattfinden" (Squarra 2019, C 2, S. 61-62). Sollte das Unwohlsein des*der Klient*In wahrgenommen werden, würde man dies problematisieren, ebenso wie den Versuch, Anliegen der Klient*Innen umzudeuten. Sollte der Coach im Rahmen des Round-Table-Gesprächs den Eindruck gewinnen, ein Coaching der Führungskraft des*der Klient*In sei (ebenfalls) angeraten, schlägt die eine Hälfte der Coaches einen erweiterten Maßnahmenkatalog vor, zu dem ein Coaching zu dritt oder ein Team-Coaching gehöre. Konträr dazu enthält sich die andere Hälfte der Coaches in dieser Situation solcher Vorschläge, weil sie sich darüber im Klaren ist, dass eine solche Argumentation ggf. als konfrontativ wahrgenommen wird und ,(..) definitiv negative Reaktionen provozieren“ (Squarra 2019, C 4, S. 104) würde. Insofern von uns die fiktive Fallgeschichte zugespitzt wird, dass die Führungskraft nicht nur Coaching-Bedarf aufweist, sondern, zudem einen konkreten Stressauslöser des*der Klient*In darstellt, können multiple und inhaltlich stark voneinander abweichende Antwortstrategien identifiziert werden: Hierzu gehört zunächst, den*die Klient*In im nachfolgenden Coaching zu ermutigen und zu befähigen, seine*ihre Führungskraft später selbst darauf anzusprechen. Daneben nennt ein Coach die Absicht, das Gespräch mit der Führungskraft zu suchen und ihr Verständnis für ihre Situation zu signalisieren, um einen Zugang zu dieser zu finden. Wiederum andere würden Maßnahmen in Form von aktivem Ansprechen bereits während der Auftragsklärung treffen, während einige Lösungsversuche in Form von Einzelgesprächen nach der Auftragsklärung, ohne Klient*In und Personaler*In, bevorzugen.

\subsection{Strategien der Auftraggeber*Innen}

\subsubsection{Fokus: Verhaltens- vs. Verhältnisprävention}

Der Frage einer psychotherapeutischen Indikation geht die eine Hälfte der befragten betrieblichen Auftraggebenden tendenziell aus dem Weg: Personaler*Innen verweisen auf Führungskräfte, diese verweisen auf Expert*Innen des Betrieblichen Gesundheitsmanagements. Dem Coach wird bei der Abgrenzung jedoch keine Rolle zugeschrieben. Die andere Hälfte der Auftraggebenden traut sich - auf Basis von Gesprächen mit dem*der Mitarbeiter*In - allerdings ein Urteil zu. Von den Mitarbeitenden erwartet man im Coaching eine konstruktive Arbeitshaltung (intrinsische Motivation). Resilienz wird zwar thematisiert, steht als Lernziel jedoch nie im Vordergrund. Die Problemanalyse solle, so die überwiegende Meinung der Auftraggebenden, auf den individuellen Arbeitsalltag des*der Mitarbeiter*In fokussieren - was nicht nur Aufgabe des Coachs sei, denn die Anpassung der Arbeitsumgebung an den*die Mitarbeiter*In sei uneingeschränkt das Ziel, eben auch des*der Auftraggeber*In. „Dann schauen wir uns diese Arbeitsumgebung genauer an und überlegen, an welcher Stelle man Abhilfe schaffen kann" (Squarra 2019, A 1, S. 152). Wenn Führung Anteil an der Problematik habe, sehen sich die Personaler*Innen in der Verantwortung, das Gespräch mit der Führungskraft zu suchen - bis hin zur Mediation. Dem Coach wird dabei allerdings keine Rolle zugedacht. Die Führungskräfte geben sich selbstreflektiert und offen für ein eigenes Coaching, um „(...) ein Gefühl dafür zu bekommen: An welchen Punkten kann ich arbeiten? Wo kann ich selbst dazu beitragen, dass bestimmte Schwierigkeiten für die Mitarbeiter entspannt werden?" (Squarra 2019, A 4, S. 221). Die Angemessenheit einer organisatorischen Problemlösung (Versetzung) wird ebenfalls benannt. 


\subsubsection{Auftragsklärung als Verhandlungssituation}

In Bezug auf die Anwesenheit der Stakeholder stellt es eher die Ausnahme dar, dass Coach und Klient*In direkt aufeinandertreffen. In den meisten Fällen werden sukzessive Varianten berichtet. Die dominante Variante der Auftragsklärung, die Auftraggebende berichten, besteht aus der Trias Personaler*In, Führungskraft und Mitarbeiter*In. Anschließend wird ein Coach gesucht, denn ,,(..) bei der Auftragsklärung (...) wissen wir noch nicht, welchen Anlass es hat, welche Ziele wir verfolgen und das heißt, dass wir noch gar nicht wissen, welcher Coach dafür gut wäre" (Squarra 2019, A 2, S. 178). Ebenfalls werden Serien von Zweiergesprächen angeführt, z.B. Führungskraft und Coach, gefolgt von Coach und Klient*In, ggf. schließt sich dann ein Dreiergespräch an. Das Verständnis der Rolle des*der Auftraggeber*In bei der Auftragsklärung erweist sich als recht divers. Einen gemeinsamen Nenner bildet die Ermittlung angemessener Zielsetzungen für das Coaching. Diese werden anschließend von der Mehrheit der Befragten in schriftliche Verträge mit dem Coach sowie dem*der Klient*In aufgenommen. Bei einer Minderheit der Befragten betreffen schriftliche Verträge lediglich Auftraggeber*In und Coach, Verträge zwischen Coach und Klient*In werden nicht erwähnt. Bei Uneinigkeit über die Zielsetzung soll das Anliegen des*der Klient*In im Vordergrund stehen, erklären die Auftraggebenden, ,denn irgendeiner, vielleicht sogar ich selbst, ist ja auf dem Holzweg, und dann lasse ich mich auch gerne von meinem Mitarbeiter belehren oder beraten, sodass man vielleicht einen anderen Weg einschlagen sollte" (Squarra 2019, A 5, S. 242). Ebenfalls würde man sich der Expertise des Coachs beugen, wenn dieser eine individualisierende Stress-Diagnose anzweifele: „(...) wenn ich einen Coach engagiere, dann weiß ich, dass der Coach gut ist, und wenn der sagt, ,hör mal, ich glaube, ich bin mir sicher zu wissen, dass es an etwas ganz anderem liegt", dann ist das auch toll“" (Squarra 2019, A 6, S. 264). Gleichwohl erwarten die Auftraggebenden nicht, dass Coaches solche Erwartungen der Auftraggebenden ungeprüft bedienen. Sie unterstreichen ihr Commitment zur Diskretion über den Coaching-Prozess. Denn ,(...), wenn der Klient das Gefühl hat, ,ich darf nichts, nur die Hälfte kommunizieren, weil mein Chef das sonst mitbekommt", da sind aus meiner Sicht die Erfolgsaussichten geringer" (Squarra 2019, A 6, S. 262).

\subsubsection{Der Round-Table: Auftragsklärung im Dreieckskontrakt}

Wenn sich der*die designierte Klient*In im Round-TableGespräch unwohl fühle, liege das meist daran, dass zu viele Personen anwesend seien. „Wir können ja dann auch sagen, wir nehmen demnächst noch einen fünften und sechsten hinzu. Also viele Köche verderben den Brei, sage ich jetzt mal“ (Squarra 2019, A 5, S. 249). Insbesondere der*die Personaler*In neige dann eher dazu, den Tisch zu verlassen, um das Gespräch nicht zu gefährden: „Als Vierter wäre ich sehr wahrscheinlich ja überflüssig“ (Squarra 2019, A 1, S. 162). Man könne in einer solchen Gesprächssituation nicht von erhöhter Offenheit und Transparenz auf allen Seiten ausgehen. „Der Klient verhält sich natürlich nicht ehrlich. Der wägt nach allen Positionen ab, egal, was ich sage, was kann jetzt mit mir passieren?"“ (Squarra 2019, A 4, S. 232). Daher sei es auch lediglich sinnvoll, abstrakte Ziele zu formulieren. Wenn beobachtet werde, dass die Anliegen des*der Klient*In manipuliert würden, würde man das Gespräch abbrechen. Sollte die Sinnhaftigkeit eines Coachings für die Führungskraft erwogen werden, erwarten Personaler*Innen, dass dies in einem separaten Gespräch thematisiert werde, und nicht konfrontativ im Round-Table-Gespräch, während Führungskräfte ihrerseits dankbar für Feedback seien und - laut eigener Aussage offen auf den Vorschlag reagieren würden.

\subsection{Strategien der Klient*Innen}

\subsubsection{Fokus: Verhaltens- vs. Verhältnisprävention}

Weder Coach noch Auftraggeber*In oder Klient*In erwägen vor Beginn eines Coachings andere Beratungsangebote oder die Sinnhaftigkeit einer Psychotherapie, berichten die Klient*Innen mehrheitlich. Diesbezüglich schwierige Phasen in der Coaching-Praxis würden von beiden Seiten als Grauzonen bezeichnet. Klient*Innen erachten eine gute Arbeitsbeziehung und angemessene Rahmenbedingungen (Zeit, Räumlichkeiten) als notwendige Voraussetzungen für Coaching. Das Resilienz-Konzept spielt in ihren Augen keine Rolle im Coaching, die Arbeitssituation und Führungsthemen hingegen stehen im Fokus. Ideen zur Verbesserung der Arbeitssituation, die im Coaching erarbeitet werden, werden in der Unternehmenspraxis jedoch kaum aufgegriffen oder umgesetzt. In diesem Zusammenhang zeigen sich ,,...) tatsächlich wenig konkrete Hilfe[n] und damit letztlich auch ein bisschen Überforderung" (Squarra 2019, K 2, S. 294). Führungsprobleme werden ggf. im Coaching analysiert und der Coach hilft dem*der Klient*In, diese in der Interaktion mit seiner*ihrer Führungskraft konstruktiv anzusprechen und Verbesserungen zu etablieren. Führungskräfte werden von den Klient*Innen in diesem Fall überwiegend als kritikfähig und änderungsbereit erlebt.

\subsubsection{Auftragsklärung als Verhandlungssituation}

Die Klient*Innen nennen die Kombination Coach und Klient*In als häufigste Variante der Auftragsklärung. Die Abwesenheit der Führungskraft bewerten sie als erleichternd, 
da ,„...) so der Fokus auch ganz klar auf meinen Themen lag und es eben nicht diesen vielleicht auch Zielkonflikt gegeben hätte" (Squarra 2019, K 3, S. 312-313). Die Dreierkombination mit internem Coach, Klient*In und Führungskraft wird an zweiter Stelle genannt und ebenfalls als angenehm bezeichnet, wenn der*die Personalentwickler*In in seiner*ihrer Rolle als interner Coach versichert, keine Informationen an die Personalabteilung zu geben. Je mehr Personen involviert sind, desto belastender erleben Klient*Innen die Auftragsklärung. Die Klient*Innen, die mit dem Coach im Zweiersetting agieren, erleben mehrheitlich, dass sie zur Klärung der Ausgangssituation beitragen sollen. Ein Drittel erlebt sich jedoch - in Anwesenheit der eigenen Führungskraft - eher in einer Statistenrolle. Vertragliche Vereinbarungen werden mit dem Coach mündlich getroffen, was den Klient*Innen ausreicht. Welche vertraglichen Vereinbarungen der Coach mit dem Unternehmen abschließt, ist den Klient*Innen überwiegend unbekannt: ,(...) kann ich die Frage eigentlich so nicht beantworten, ich weiß nicht, was da zwischen Coach und Firma läuft" (Squarra 2019, K 6, S. 358). Die überwiegende Mehrheit der Klient*Innen kann ihre Anliegen vortragen und zum Arbeitsgegenstand machen. Lediglich in einem Fall wird erlebt, dass der Coach sich die Zielsetzung und Führungsdelegation des Unternehmens zueigen macht. Die Vertraulichkeit, berichten die Klient*Innen, wird ihres Erachtens nach in jedem Fall gewahrt.

\subsubsection{Der Round-Table: Auftragsklärung im Dreieckskontrakt}

Die Teilnahme am Round-Table-Gespräch empfinden alle Klient*Innen als unangenehm, insbesondere bei Anwesenheit des*der Personaler*In: „Er hat mich angeschaut und gesagt: ,Leistung ist Arbeit pro Zeiteinheit.' Das hatte jetzt für den Coaching-Prozess, nun, überhaupt keinen (...) Mehrwert" (Squarra 2019, K 4, S. 325). Es wird zudem befürchtet, dass ,(...) der Personaler das dann vielleicht noch mit in die Personalakte nimmt (lacht)“ (Squarra 2019, K 5, S. 346). Die Gesprächsatmosphäre wird als gekünstelt und wenig offen erlebt - insbesondere, wenn das Verhältnis zur Führungskraft angespannt ist. Ziele werden daher eher abstrakt und pro forma benannt. Erleben die Klient*Innen allerdings, dass ihre Anliegen umgedeutet werden, würde die Mehrheit dies offen ansprechen. Sollte ein Coach in dieser Situation ein Coaching für den*die Auftraggeber*In ansprechen, würde dies nach Meinung der Klient*Innen keine offenen Ohren finden. Denn für die Führungskraft „(...) könnte das sehr schnell einen Gesichtsverlust bedeuten“ (Squarra 2019, K 2, S. 304).

\subsection{Vergleichende Betrachtung}

\subsubsection{Fokus: Verhaltens- vs. Verhältnisprävention}

Sowohl Coaches als auch Auftraggebende diagnostizieren, indem ein potenziell psychotherapeutischer Bedarf in den meisten Fällen zugunsten von Coaching ausgeschlossen wird. Die Coaches berufen sich auf ihre Kompetenz. Die Auftraggebenden wiederum verweisen auf ihre eigene Kompetenz oder auf die anderer Akteur*Innen (Führungskräfte, BGM), aber interessanterweise nicht auf den Coach. Die Klient*Innen schließlich berichten, dass dieses Thema im Auftragsklärungsgespräch gar keine Rolle spielte. Die Problemanalyse beinhaltet für alle Stakeholder - neben der Rolle des*der Mitarbeiter*In - die Betrachtung des Arbeitskontextes sowie der Führung. Die Bearbeitung dieser Problembereiche findet offenbar weitgehend nur im Coaching selbst statt. Sie startet damit, dass eine eigenständige Problemanalyse erfolgt, dessen sind sich auch die Auftraggebenden bewusst. $\mathrm{Ob}$ und inwieweit eine solche Bearbeitung jenseits des Coaching-Prozesses erfolgt, bleibt jedoch offen. Lösungsideen für den Arbeitskontext werden im Coaching weniger verfolgt, Coaches wollen den Führungskräften nicht in deren Aufgabengebiet hineinregieren. Lösungsideen für die Führungssituation werden gleichwohl entwickelt sowie eine Kompetenzentwicklung der Klient*Innen forciert, zu der neben verhaltensbasierten auch emotionsbasierte Copingstrategien gehören. Coaches fördern Änderungen eher indirekt, indem sie ihre Klient*Innen im Sinne einer Hilfe zur Selbsthilfe befähigen, diese mit der Führungskraft zu besprechen. Klient*Innen bezeichnen die Reaktion der Führungskräfte auf ihre Vorschläge überwiegend als nachdenklich und veränderungswillig. Teilweise sucht der Coach auch das Gespräch mit der Führungskraft, um sehr behutsam Verständnis für den*die Mitarbeiter*In zu entwickeln. Einige Auftraggebende erklären, dass sie dankbar für Kritik seien, da sie ihr Führungsverhalten nur so stetig verbessern könnten. Klient*Innen erleben jedoch mehrheitlich keine Veränderungen ihrer Arbeitssituation nach solchen Gesprächen.

\subsubsection{Auftragsklärung als Verhandlungssituation}

In allen drei Gruppen lassen sich zehn verschiedene Konstellationen der Beteiligten in der Auftragsklärung ermitteln. Die Lage lässt sich daher als recht heterogen beschreiben. Die von Coaches sowie Klient*Innen meistgenannte Konstellation stellt deren gemeinsames Auftragsklärungsgespräch dar, was beide als angenehm erleben. Auftraggebende nennen am häufigsten die Dreierkonstellation Personaler*In, Führungskraft und Klient*In zur Auftragsklärung. Begründet wird dies mit der Notwendigkeit, den CoachingBedarf zu spezifizieren, um anschließend einen geeigneten 
Coach suchen zu können. Die Kompetenz hierzu wird insbesondere dem*der Personaler*In attribuiert. Das Anliegen der Auftraggebenden im Klärungsgespräch mit dem Coach besteht in einer Zieldefinition. Die Coaches heben insbesondere die Notwendigkeit hervor, den Coaching-Prozess im geschützten Rahmen durchführen zu wollen, ohne dass sich die Auftraggebenden einmischen. Coaches berichten von Versuchen der Führungsdelegation und von eigenen Tendenzen zur Solidarisierung mit dem*der Klient*In. Klient*Innen können ihre Anliegen überwiegend einbringen, in die Vertragsgestaltung zwischen dem Coach und dem Unternehmen werden sie jedoch nicht einbezogen. Neben diesen dominanten Varianten werden auch diverse sukzessive bilaterale Gesprächskonstellationen berichtet.

\subsubsection{Der Round-Table: Auftragsklärung im Dreieckskontrakt}

Das Round-Table-Gespräch (Dreieckskontrakt) wird von allen Beteiligten als wenig hilfreich erlebt, da es nicht gelinge, die nötige Offenheit herzustellen. Eine Weitung der Perspektive in Richtung Arbeitsgestaltung oder Führung unterbleibe oder würde keine Konsequenzen haben. Zielbeschreibungen blieben folglich recht abstrakt. Klient*Innen gelinge es dabei nur teilweise, ihre Anliegen einzubringen, für sie sind andere Settings vor diesem Kontext zielführender.

\section{Diskussion}

Aus der Zusammenschau der Perspektiven und der gegenseitigen argumentativen Verweise der befragten Stakeholder ergeben sich Antworten auf die Forschungsfragen, die die aufgestellten Hypothesen in den meisten Fällen unterstützen und darüber hinaus die Sachverhalte weiter erhellen.

\subsection{Fokus: Verhaltens- vs. Verhältnisprävention}

Es muss angenommen werden, dass Coaching auch für unpassende Problemlagen wie behandlungsbedürftige psychische Störungen eingesetzt wird. Dies ergibt sich daraus, dass keiner der Stakeholder in der Lage ist, solche Indikationen zu diagnostizieren; es fehlt hier allseitig schlicht an Kompetenz. Die Bearbeitung unpassender Problemlagen durch Coaching erweist sich jedoch für die involvierten Stakeholder als funktionale Strategie: Den Coaches erschließt es ein zusätzliches Klient*Innensegment, worauf schon Werner und Webers (2016) hinweisen. Den Klient*Innen erspart es eine befürchtete Stigmatisierung, die mit einer Psychotherapie-Indikation verbunden sein kann (BAuA 2017). Zugleich verschafft es Klient*Innen und ebenfalls den Unternehmen die Hoffnung, Problemlagen bearbeiten zu können, die im vermeintlichen Graubereich zwischen Psychotherapie und Coaching liegen und die ansonsten - u.a. wegen nicht unerheblichen Wartezeiten im psychotherapeutischen Versorgungssystem - länger persistieren. Coaching erweist sich damit als ein niedrigschwelliges Angebot - auch im Sinne des Betrieblichen Gesundheitsmanagements (BGM). Als Gefahr erscheint dabei allerdings die in der Regel unzureichende Qualifikation der Coaches. Eventuelle diagnostische Zweifel kommunizieren Coaches gegenüber dem*der Auftraggeber*In offenbar selten, da sie einen Widerspruch als riskant für das eigene Auftragsverhältnis empfinden. Ein Coaching verhindert in solchen Fällen ggf. das Aufsuchen angemessener Hilfsangebote und kann zu Verschlimmerung und Chronifizierung führen. Allerdings ergibt sich an dieser Stelle die Schwierigkeit, eine sinnvolle Abgrenzung in der Praxis zu etablieren. Eine potenzielle Verbesserung könnte in der spezifischen Qualifizierung von Coaches bestehen, die von Weiterbildungsinstituten in die Hand genommen und von Verbänden durch Standards unterstützt werden sollte. Allerdings sind auch die Unternehmen in der Pflicht, wie nicht zuletzt das Arbeitsschutzgesetz (Gefährdungsbeurteilung psychischer Belastungen) fordert.

Coaching stellt - im Lichte dieser Untersuchung primär eine verhaltensbezogene Intervention dar. Wenn auch die Verhältnisse, also die Arbeitsbedingungen und die Führungssituation, von allen befragten Stakeholdern als wichtig bezeichnet werden, so findet deren Exploration weitestgehend lediglich im Coaching-Prozess von Coach und Klient*Innen statt. Verbesserungsvorschläge, die diese Verhältnisse betreffen, finden aus dem vertraulichen Zweiergespräch eher indirekt und dosiert ihren Weg in die betriebliche Arena zurück. Unbestritten können im Coaching-Prozess diverse verhaltensbezogene Copingstrategien entwickelt und umgesetzt werden. Dies mag man als Kompetenzentwicklung und Empowerment des*der Klient*In bezeichnen und begrüßen. Veränderungen der Arbeitsbedingungen werden von Coaches aber eher ausgeklammert. Veränderungen der Führungssituation können auf diesem Wege nur vermittelt über den*die Klient*In - oder in der behutsamen Direktansprache, den eine Untergruppe der Coaches wählt, - fruchtbar werden. Doch Führung ist per se eine zweiseitige Relation. Es wird deutlich, dass sich hier eine Seite strukturell der Verantwortung entziehen kann - auch wenn die Führungskräfte, darauf angesprochen, anderes antworten. Letzteres wäre, so die Hypothese, ein wohlfeiles Lippenbekenntnis. Die betriebliche Machtposition schützt die Führungskraft einerseits gegenüber dem*der Mitarbeiter*In (Hierarchiegefälle), andererseits als Auftraggeber*In gegenüber dem Coach (Einkäufer*In). Dies schließt nicht aus, dass selbstkritische und änderungsbereite Führungskräfte solche Impulse aufgreifen und umsetzen. 


\subsection{Auftragsklärung als Verhandlungssituation}

Schwertl, der das Dilemma zweier Verträge im Coaching thematisiert, rät, der Coach solle sich als „Dialogförderer für das ganze System“ (Schwertl 2015, S. 50) anbieten. Um den verhaltenspräventiven Bias zu vermeiden, wäre es weiterführend sinnvoll, Erkenntnisse der Bildungstransferforschung zu berücksichtigen. Nach Baldwin und Ford (1988) entscheiden drei Faktoren über den Erfolg einer Weiterbildungsmaßnahme: Neben den Teilnehmerbedingungen (Motivation etc.) sind dies Trainingsbedingungen (bspw. die Form des Coachings) sowie die Arbeitsumgebung (Förderung durch das Management und unterstützende Organisationskultur). Dieses Konzept hat sich in der Forschung allgemein (Kauffeld et al. 2012), aber auch in der CoachingForschung (Bickerich und Michel 2018) bewährt. Unternehmen bieten sich damit zwei Hebel zur Verbesserung der Situation an:

- Coaching-Bedingungen: Das Zweiersetting lässt sich durch Shadowing (Begleitung des*der Klient*In am Arbeitsplatz durch den Coach) und weitere systemische Maßnahmen wie Führungsfeedback oder Teamworkshops öffnen. Damit entsteht mehr Transparenz für die betriebliche Wirklichkeit im Coaching, aber auch mehr Transparenz für das Coaching im Betrieb.

- Arbeitsumgebung: Das Management kann das Coaching unterstützen, indem Daten und Materialien (Arbeitsgestaltung, Führung) sowie die Mitarbeit offensiv angeboten werden. Damit würden auch wichtige kulturelle Signale in die Organisation kommuniziert - dass nicht lediglich am*an der Mitarbeiter*In „kuriert“ wird, sondern man an einer systemischen Verbesserung interessiert ist.

Beide strategische Optionen werden in dieser Untersuchung weniger sichtbar. Stattdessen erfährt man, dass sich einige Coaches nicht trauen, das Setting zu ändern, oder dass befürchtet wird, dass solche Vorschläge zum Auftragsverlust führen. Klient*Innen berichten, dass sie zwar Nachdenklichkeit hinsichtlich des Führungsverhaltens bewirken, letztlich aber kaum Veränderungen ihrer Arbeitssituation erreichen können.

Es ist folglich - sensu Schwertl (2015) - ratsam, dass Coaches ein ganzheitliches Coaching-Verständnis und die daraus abgeleiteten Konsequenzen schon im Rahmen der Auftragsklärung darlegen und ein entsprechendes Commitment vom Unternehmen einfordern. So wird die Rolle der Bedingungsvariablen neben den Personenvariablen schon zu Beginn als fester Bestandteil professionellen Coachings sichtbar und verständlich, und der*die Auftraggeber*In muss sich dazu positionieren. - Ein Problem lässt sich so allerdings nicht lösen: die Auftragslage von Coaches.
Auf das Setting der Auftragsklärung können, so zeigen die Ergebnisse dieser Studie, sowohl Coaches als auch Klient*Innen wenig Einfluss nehmen, während Auftraggebende sämtliche Details, von der Anwesenheit bis zur Gesprächsreihenfolge, festlegen. So wurden insgesamt zehn verschiedene Varianten und Reihenfolgen berichtet. Aus der Datenlage entsteht der Eindruck, dass jedes Unternehmen willkürlich festlegt, welche Akteur*Innen zu welchem Zeitpunkt bei der Auftragsklärung anwesend sein dürfen und in wie vielen Schritten diese erfolgt. Daher kann angenommen werden, dass in der Praxis noch mehr Varianten existieren, weshalb sich hier, langfristig betrachtet, eine Vereinheitlichung für unternehmensbezogene Auftragsklärungen empfehlen würde.

\subsection{Der Round-Table: Auftragsklärung im Dreieckskontrakt}

Bei der Rollenverteilung der einzelnen Stakeholder zeigt sich insgesamt die Tendenz, dass Klient*Innen eine deutlich passivere Rolle einnehmen, sobald Auftraggebende anwesend sind. Der Dreieckskontrakt, der in der Literatur zur Lösung dieses Problems vorgeschlagen wird und offensichtlich in einigen Unternehmen seit vielen Jahren praktiziert wird (Limpächer und Limpächer 2015), erscheint vor dem Hintergrund dieser Studie als weniger geeignet. Er scheint zu einem formalisierten, oberflächlichen Gespräch $\mathrm{zu}$ führen, das weniger Vertrauen aufbaut, als Befürchtungen weckt. Schon Dehner (2013) hat dies gemutmaßt und sein Vorschlag, stattdessen bilaterale Gespräche zu führen, wird auch von den Teilnehmenden dieser Studie präferiert.

Insofern Coaches mit dem Fall konfrontiert werden, dass die Führungskraft ein Coaching benötigt und sich im Rahmen der Auftragsklärung als Stressauslöser des*der Klient*In entpuppt, ist das Strategieverhalten stark heterogen. Demnach scheint in schwierigen Situationen mehr Improvisation als durchdachte Handlungsweisen zu erfolgen. Es kann vermutet werden, dass bisher noch keine optimalen Lösungsstrategien für solch komplexe Situationen in der Auftragsklärung existieren.

Die Befürchtungen der Klient*Innen bezüglich der Rolle des*der Personaler*In im Vierecksvertrag zeigen, dass dieser nicht nur positiv als Anwalt*Anwältin des*der Mitarbeiter*In gesehen wird, sondern auch seine*ihre sanktionierende Rolle wahrgenommen wird. Schon Backhausen und Thommen (2017) hinterfragen die Unabhängigkeit der Personalabteilung sowie -entwicklung im gesamten Coaching-Prozess. Kühl (2008) betont die Konkurrenz zwischen Führungskraft und Personalabteilung: Die Personaler*Innen regulieren die Anbieterseite durch die Einrichtung von Coach-Pools. Zugleich positionieren sie sich auf diese Weise anspruchsvoll und mächtig gegenüber den eigenen Führungskräften als „Gatekeeper“. Diese Konkurrenz 
kann sich auch im Round-Table-Gespräch offenbaren. Dies würde reserviertes Verhalten der Führungskräfte erklären können, welche - wie auch die Klient*Innen - geringe eigene Offenheit im Rahmen des Vierersettings äußern.

\section{Kritische Würdigung}

Die Stichprobe $(N=18)$ ist klein, Selektionseffekte können nicht ausgeschlossen, Repräsentativität kann nicht reklamiert werden. Doch die gewählte Methode eines qualitativen Designs ermöglicht einen Einblick in das Erleben der Stakeholder - und die gegenseitige argumentative Bezugnahme. So wird strategisches Verhalten verständlich. Diese geschlussfolgerten Annahmen müssten in weiterer Forschung akribisch geprüft werden, da nur auf diese Weise die sich in dieser Studie offenbarenden Widersprüche konkreter untersucht werden können. So unterbleibt eine Argumentation der Abhängigkeit der Auftragsklärungsstrategie vom Unternehmenskulturtyp. Ein weiterer Forschungsansatz könnte in einer Kreuzvalidierung mit einer neuen Stichprobe bestehen. Ebenfalls könnte die ökologische Validität durch einen Wechsel der Befragungsrichtung verbessert werden: In der vorliegenden Arbeit wurden die Expert*Innen frei zu ihrem Erleben der Auftragsklärung befragt. In einer neuen Studie könnten Expert*Innen mit einem konkreten Vorgehensmodell (Best Practice) konfrontiert und um Kommentierung gebeten werden. Demselben Anliegen würde ein Ansatz mit der Critical-Incidence-Methode dienen (Flanagan 1954). Ein Verfahren zur kommunikativen Validierung bestünde im Einsatz der Delphi-Methode, bei der es sich um ein mehrstufiges Expert*InnenBefragungsverfahren handelt, das sich bei Fragen der Personalentwicklung schon bewährt hat (Schermuly et al. 2012). Auf diese Weise ließen sich im besten Falle Widersprüchlichkeiten und offene Fragen, die die vorliegende Studie nicht erhellen konnte, weiter erforschen.

Eine Möglichkeit, die Güte der Ergebnisse zu verbessern, stellt die Analyse und Auswertung durch verschiedene Forscher*Innen (Investigator Triangulation) dar (Flick 2011). Hierdurch ließe sich insbesondere die für die qualitative Inhaltsanalyse relevante Intercoderreliabilität (Mayring 2015) erhöhen. Die Kombination mit quantitativen Methoden im Sinne eines „Mixed-Methods“-Ansatzes kann zukünftig ein weiteres und tieferes Verständnis des Themas fördern.

Darüber hinaus werfen die Ergebnisse dieser Studie weitere Fragen zu bisher wenig beleuchteten Themen auf. So wird in dieser Studie der*die Klient*In als schwächstes Glied in der Kette der Stakeholder und als schützenswert dargestellt. Diese Vorannahme muss man nicht teilen. Denkbar ist, dass Klient*Innen das Coaching auch für eigene Zwecke nutzen, um sich bspw. strategische Vorteile im innerbetrieblichen Wettbewerb zu verschaffen, sich gegen- über Kolleg*Innen oder der Führungskraft in eine bessere Position zu bringen (Blickle 2004). Solidarisierungsvorwürfe gegenüber dem Coach, er*sie fraternisiere leichtfertig mit dem*der Klient*In (Schmid und Hipp 2003), erscheinen dann in einem anderen Licht, nämlich als mögliche Delegationsversuche: Der Coach soll stellvertretend Probleme des*der Klient*In lösen.

\section{Fazit und Ausblick}

Die vorliegende Arbeit präsentiert Ergebnisse über Verhalten und Erleben bei der Auftragsklärung im Unternehmenskontext und liefert somit einen neuen Beitrag zum Forschungsbereich des Coachings. Aus multiperspektivischer Sicht - um den Titel der Arbeit aufzugreifen - lässt sich die Forschungsfrage ,Welche Strategien verfolgen Coaches, Auftraggebende und Klient*Innen in schwierigen Situationen bei der Auftragsklärung und wie erleben sie diese?" inhaltlich nicht eindeutig beantworten. Dies ist damit zu begründen, dass die Stakeholder sich teilweise widersprechen. Aussagen im betrieblichen Kontext sind immer auch politische Statements. Am aufschlussreichsten ist daher die Betrachtung der Widersprüche. Insgesamt spiegeln diese eine Verhandlungssituation, in der explizite und implizite Interessen eine Rolle spielen, wider.

Aus den gewonnenen Daten entsteht der Eindruck, dass Coaches und Klient*Innen ihre Strategien unter Vorsicht ausführen, da sie dem*der Auftraggeber*In als höherer Instanz Vergeltungsmaßnahmen zutrauen. Bei Coaches scheint diese Vermutung jedoch deutlich ausgeprägter zu sein. Dies hat zur Konsequenz, dass diese zum Teil verdeckte Strategien in den Coaching-Sitzungen verfolgen, um in keinen Konflikt zum*zur Auftraggeber*In zu geraten. Auftraggebende selbst stellen sich wiederum kritikfähig und kompromissbereit dar, sodass diese zukünftig konkreter auf die Ernsthaftigkeit ihrer als kooperativ dargestellten Strategien untersucht werden müssten. Jedoch bleibt ebenso das Strategieverhalten der Coaches fragwürdig, da die Aussagen der Klient*Innen vermehrt auf Indizien für Problemindividualisierungen hindeuten. Dadurch kann die Einsatzbereitschaft der Coaches im Rahmen konfliktbehafteter Situationen angezweifelt werden. Die Klient*Innen scheinen sich in der hilflosesten Position zu befinden, da ihre Verhaltensweisen durch Coach und Auftraggeber*In weitestgehend determiniert werden: Die eigene Rollenausübung, Vertragsgestaltung, Setting der Auftragsklärung oder bestimmte Maßnahmen zur Problemlösung sind nur einige Bespiele, bei welchen Klient*Innen sich fast ausschließlich nach den Vorgaben der übrigen Stakeholder richten. 
Interessenkonflikt A. Squarra und T. Webers geben an, dass kein Interessenkonflikt besteht.

Open Access Dieser Artikel wird unter der Creative Commons Namensnennung 4.0 International Lizenz veröffentlicht, welche die Nutzung, Vervielfältigung, Bearbeitung, Verbreitung und Wiedergabe in jeglichem Medium und Format erlaubt, sofern Sie den/die ursprünglichen Autor(en) und die Quelle ordnungsgemäß nennen, einen Link zur Creative Commons Lizenz beifügen und angeben, ob Änderungen vorgenommen wurden.

Die in diesem Artikel enthaltenen Bilder und sonstiges Drittmaterial unterliegen ebenfalls der genannten Creative Commons Lizenz, sofern sich aus der Abbildungslegende nichts anderes ergibt. Sofern das betreffende Material nicht unter der genannten Creative Commons Lizenz steht und die betreffende Handlung nicht nach gesetzlichen Vorschriften erlaubt ist, ist für die oben aufgeführten Weiterverwendungen des Materials die Einwilligung des jeweiligen Rechteinhabers einzuholen.

Weitere Details zur Lizenz entnehmen Sie bitte der Lizenzinformation auf http://creativecommons.org/licenses/by/4.0/deed.de.

\section{Literatur}

Bachmann, T. (2016). Implizite Theorien über Lernen und Veränderungen durch Coaching in Organisationen - Eine empirische Untersuchung. Organisationsberatung - Supervision - Coaching, 23(3), 231-253. https://doi.org/10.1007/s11613-016-0467-z.

Backhausen, W., \& Thommen, J.-P. (2017). Coaching. Durch systemisches Denken zu innovativer Personalentwicklung. Wiesbaden: Springer.

Baldwin, T.T., \& Ford, J.K. (1988). Transfer of training: a review and directions for future research. Personnel Psychology, 41(1), 63-105. https://doi.org/10.1111/j.1744-6570.1988.tb00632.x.

Bamberg, E., \& Vincent-Höper, S. (2018). Gesundheit im Coaching. In S. Greif, H. Möller \& W. Scholl (Hrsg.), Handbuch Schlüsselkonzepte im Coaching (S. 235-243). Berlin: Springer. https://doi.org/ 10.1007/978-3-662-49483-7_20.

BAu, A. (Hrsg.). (2017). Psychische Gesundheit in der Arbeitswelt - Wissenschaftliche Standortbestimmung. Dortmund: Bundesanstalt für Arbeitsschutz und Arbeitsmedizin.

Bickerich, K., \& Michel, A. (2018). Change-Prozesse als Anwendungsfeld im Coaching. In S. Greif, H. Möller \& W. Scholl (Hrsg.), Handbuch Schlüsselkonzepte im Coaching (S. 75-83). Berlin: Springer. https://doi.org/10.1007/978-3-662-49483-7_5.

Blickle, G. (2004). Einfluss ausüben, Ziele verwirklichen. Ein Überblick über Einflusstaktiken in Organisationen und ihre situationsspezifischen Wirkmechanismen. Personalführung, 37(6), 58-70.

DBVC (Hrsg.). (2013). Zusammenfassung: Coaching-Marktanalyse 2013. Ergebnisse der 3. Marburger Coaching-Studie 2013. Osnabrück: DBVC.

Dehner, U. (2013). Häufig gestellte Fragen. Coaching Magazin, 6(2), 35.

Dresing, T., \& Pehl, T. (2015). Praxisbuch Interview, Transkription und Analyse. Anleitungen und Regelsysteme für qualitativ Forschende (6. Aufl.). Marburg: Eigenverlag.

English, F. (1975). The three-cornered contract. Transactional Analysis Journal, 5(4), 383-384. https://doi.org/10.1177/03621537750050 0413.

Fatzer, G., \& Webers, T. (2008). Coaching darf sich nicht degradieren lassen. Der Coach muss weit mehr als nur Beichtvater sein. Coaching-Magazin, 1(3), 12-17.

Fischer, H.-R., von Schlippe, A., \& Borst, U. (2014). Über Erwartungen und Aufträge in Therapie und Beratung. Familiendynamik, 39(3), 260-263.

Flanagan, J.C. (1954). The critical incident technique. Psychological Bulletin, 51(4), 327-358. https://doi.org/10.1037/h0061470.

Flick, U. (2011). Triangulation. Eine Einführung. Wiesbaden: VS.
Franke, F., Ducki, A., \& Felfe, J. (2015). Gesundheitsförderliche Führung. In J. Felfe (Hrsg.), Trends der psychologischen Führungsforschung (S. 253-264). Göttingen: Hogrefe.

Greif, S., \& Bertino, M. (2018). Burnout: Merkmale und Prävention im Coaching. In S. Greif, H. Möller \& W. Scholl (Hrsg.), Handbuch Schlüsselkonzepte im Coaching (S. 53-62). Berlin: Springer. https://doi.org/10.1007/978-3-662-49483-7 4.

Greif, S., Möller, H., \& Scholl, W. (2018). Coachingdefinitionen und -konzepte. In S. Greif, H. Möller \& W. Scholl (Hrsg.), Handbuch Schlüsselkonzepte im Coaching (S. 1-9). Berlin: Springer. https:// doi.org/10.1007/978-3-662-49483-7_7.

Helfferich, C. (2014). Leitfaden- und Experteninterviews. In N. Baur \& J. Blasius (Hrsg.), Handbuch Methoden der empirischen Sozialforschung (S. 559-575). Wiesbaden: Springer.

Ilmarinen, J., Tuomi, K., \& Seitsamo, J. (2005). New dimensions of work ability. Assessment and promotion of work ability, health and well-being of ageing workers. Proceedings of 2nd International Symposium on Work Ability, Verona, 18.-20. October 2004. International Congress Series, 1280 (0). (S. 3-7). https://doi.org/ 10.1016/j.ics.2005.02.060.

Kauffeld, S., Lorenzo, G., \& Weisweiler, S. (2012). Wann wird Weiterbildung nachhaltig? - Erfolg und Erfolgsfaktoren beim Lerntransfer. PERSONALquarterly, 64(2), 10-15.

Kühl, S. (2008). Die nur fast gelingende Schließung des Personalentwicklungszyklus. Organisationsberatung - Supervision Coaching, 15(2), 137-155. https://doi.org/10.1007/s11613-0080075-7.

Kühl, S. (2018). Rollen als Grundlagenthema im Coaching. In S. Greif, H. Möller \& W. Scholl (Hrsg.), Handbuch Schlüsselkonzepte im Coaching (S. 495-502). Berlin: Springer. https://doi.org/10.1007/ 978-3-662-49483-7 57.

Limmer, A., \& Schütz, A. (2018). Resilienz - Modewelle oder Paradigmenwechsel in Prävention und Coaching? In S. Greif, H. Möller \& W. Scholl (Hrsg.), Handbuch Schlüsselkonzepte im Coaching (S. 485-493). Berlin: Springer. https://doi.org/10.1007/978-3662-49483-7_55.

Limpächer, S., \& Limpächer, M. (2003). Dreiecksverträge im Coaching: Vertraulichkeit versus Vertraglichkeit? Wirtschaftspsychologie aktuell, 10(3), 61-66.

Limpächer, S., \& Limpächer, M. (2015). Internes Coaching mit Round-Table-Vertragsarbeit. In A. Dollinger \& S. Limpächer (Hrsg.), Internes Coaching. Praxisberichte, Prozesse, Methoden (S. 248-267). Weinheim: Beltz.

Löwer-Hirsch (2017). Inszenierungen von Macht und Ohnmacht bei der Auftragsklärung und im Erstkontakt. Organisationsberatung - Supervision - Coaching, 24(3), 313-322. https://doi.org/10. 1007/s11613-017-0514-4.

Mayring, P. (2015). Qualitative Inhaltsanalyse. Grundlagen und Techniken (12. Aufl.). Weinheim: Beltz.

Montano, D., Reeske, A., Franke, F., \& Hüffmeier, J. (2017). Leadership, followers' mental health and job performance in organizations: a comprehensive meta-analysis from an occupational health perspective. Journal of Organizational Behavior, 38(3), 327-350. https://doi.org/10.1002/job.2124.

Pühl, H. (2006). Verordnetes Coaching - und Möglichkeiten und Grenzen zur Organisationsentwicklung. Organisationsberatung - Supervision - Coaching, 13(2), 193-198. https://doi.org/10.1007/ s11613-006-0026-0.

Pühl, H. (2008). Auftragsklärung: Interventionen auf dem Wege zur Konstituierung eines Beratungssystems. Organisationsberatung Supervision - Coaching, 15(3), 341-352. https://doi.org/10.1007/ s11613-008-0090-8.

Rauen, C. (2014). Coaching. Bd. 3. Göttingen: Hogrefe.

Schermuly, C.C. (2016). Negative Effekte von Coaching für Klienten Definition, Häufigkeiten, Kategorien und Ursachen. In C. Triebel, J. Heller, B. Hauser \& A. Koch (Hrsg.), Qualität im Coaching. Denkanstöße und neue Ansätze: Wie Coaching mehr Wirkung und 
Klientenzufriedenheit bringt (S. 205-214). Heidelberg: Springer. https://doi.org/10.1007/978-3-662-49058-7_17.

Schermuly, C.C., Schröder, T., Nachtwei, J., Kauffeld, S., \& Gläs, K. (2012). Die Zukunft der Personalentwicklung. Eine Delphi-Studie. Zeitschrift für Arbeits- und Organisationspsychologie, 56(3), 111-122. https://doi.org/10.1026/0932-4089/a000078.

Schmid, B., \& Hipp, J. (2003). Kontraktgestaltung im Coaching. Organisationsberatung - Supervision - Coaching, 10(3), 255-263. https://doi.org/10.1007/s11613-003-0029-z.

Schwertl, W. (2015). Ein Coach muss verschwiegen sein, aber. wirtschaft + weiterbildung, 27(2), 48-50.

Schyns, B., \& Schilling, J. (2013). How bad are the effects of bad leaders? A meta-analysis of destructive leadership and its outcomes. The Leadership Quarterly, 24(1), 138-158. https://doi.org/10. 1016/j.leaqua.2012.09.001.

Squarra, A. (2019). Auftragsklärung im Coaching - eine Multistakeholder-Perspektive (Unveröffentlichte Masterarbeit). Köln: Hochschule Fresenius.

Ulich, E. (2011). Arbeitspsychologie. Bd. 7. Stuttgart: Schäffer-Poeschel.

Veith, H., \& Veith, T. (2014). Rollenkompetenz als Coach - weit mehr als Handwerkszeug. In A. Ryba, D. Pauw, D. Ginati \& S. Rietmann (Hrsg.), Professionell coachen. Das Methodenbuch: Erfah- rungswissen und Interventionstechniken von 50 Coachingexperten (S. 53-69). Weinheim: Beltz.

WAI-Netzwerk (Hrsg.). (2015). WAI-Manual. Anwendung des WorkAbility Index. Wuppertal: Institut für Sicherheitstechnik, Bergische Universität Wuppertal.

Webers, T., \& Zickermann, R. (2019). Training vs. Coaching - über Aporien in der Professionalisierungsdiskussion. Coaching Theorie \& Praxis, 5(1), 35-50. https://doi.org/10.1365/s40896-01900030-z.

Werner, F., \& Webers, T. (2016). Erkennen Coaches einen Psychotherapiebedarf ihrer Klienten? Coaching Magazin, 9(1), 50-54.

Wieland, R., Winizuk, S., \& Hammes, M. (2009). Führung und Arbeitsgestaltung - Warum gute Führung allein nicht gesund macht. Arbeit - Zeitschrift Für Arbeitsforschung, Arbeitsgestaltung und Arbeitspolitik, 18(4), 282-297. https://doi.org/10.1515/arbeit2009-0404.

Wolff, U. (2012). Anwendungsbereiche. In DBVC (Hrsg.), Leitlinien und Empfehlungen für die Entwicklung von Coaching als Profession. Kompendium mit den Professionsstandards des DBVC (Bd. 4, S. 21-26). Osnabrück: Deutscher Bundesverband Coaching.

Publisher's Note Springer Nature remains neutral with regard to jurisdictional claims in published maps and institutional affiliations. 